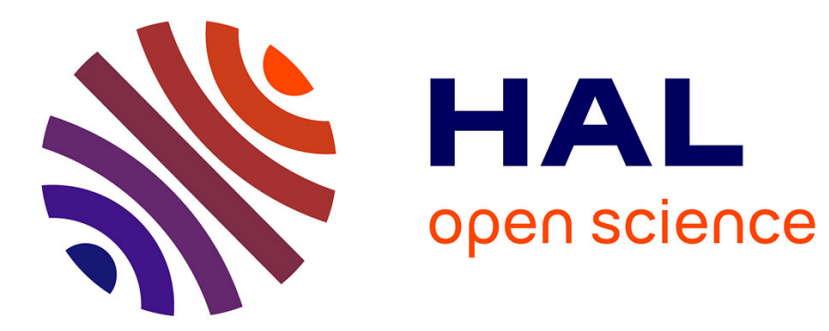

\title{
Isotropic-to-nematic transition in wormlike micelles under shear
}

Jean-François Berret, Denis Roux, Grégoire Porte

\section{To cite this version:}

Jean-François Berret, Denis Roux, Grégoire Porte. Isotropic-to-nematic transition in wormlike micelles under shear. Journal de Physique II, 1994, 4 (8), pp.1261-1279. 10.1051/jp2:1994198 . jpa-00248041

\section{HAL Id: jpa-00248041 https://hal.science/jpa-00248041}

Submitted on 1 Jan 1994

HAL is a multi-disciplinary open access archive for the deposit and dissemination of scientific research documents, whether they are published or not. The documents may come from teaching and research institutions in France or abroad, or from public or private research centers.
L'archive ouverte pluridisciplinaire HAL, est destinée au dépôt et à la diffusion de documents scientifiques de niveau recherche, publiés ou non, émanant des établissements d'enseignement et de recherche français ou étrangers, des laboratoires publics ou privés. 
Classification

Physics Abstracts

$64.70-61.30 \mathrm{E}-46.30 \mathrm{~J}$

\title{
Isotropic-to-nematic transition in wormlike micelles under shear
}

\author{
Jean-François Berret, Denis C. Roux and Grégoire Porte \\ Groupe de Dynamique des Phases Condensées (*), Université de Montpellier II, \\ 34095 Montpellier Cedex 05, France
}

(Recetved 20 January 1994, revised 13 April 1994, accepted II May 1994)

\begin{abstract}
We report on the linear and nonlinear rheology of surfactant solutions of elongated wormlike micelles. The surfactant solutions placed under scrutıny are made of cetylpyridinium chloride $\left(\mathrm{CP}^{+}, \mathrm{Cl}^{-}\right)$and sodium salıcylate $\left(\mathrm{Na}^{+}, \mathrm{Sal}^{-}\right)$diluted in $0.5 \mathrm{M} \mathrm{NaCl}$-brine. Both semidilute and concentrated regimes of entangled micelles were investigated. Rheological experiments were performed at ambient temperature $\left(T=25^{\circ} \mathrm{C}\right)$ for surfactant concentrations $\phi=1 \%-30 \%$. When submitted to a steady shear high enough (for shear rate $\dot{\gamma}$ typically higher than $1-10 \mathrm{~s}^{-1}$ ) the solutions of wormlike micelles exhibit a first-order isotropic-to-nematic transition for all surfactant concentrations $\phi \geqslant 6 \%$. The transition is characterized by a true plateau in the shear rate dependence of the shear stress $\sigma(\dot{\gamma})$. For $\dot{\gamma}$ above the transition rate $\dot{\gamma}_{1 / N}, \sigma$ remains constant at $\sigma_{1 / \mathbb{N}}$. In the concentrated regime, the transition is clearly first-order. However, the first-order character weakens upon increasing dilution, suggesting that at some critical concentration $\phi_{\mathrm{c}}$ it becomes second-order. Below $\phi_{\mathrm{c}}$, the transition ceases to occur: the $\sigma(\dot{\gamma})$-behavior rather indicates a progressive and homogeneous orientation of the micelles throughout the sample. Moreover, in the two-phase domain (where both isotropic and nematic phases coexist) a characteristic transient behavior of the shear stress $\sigma(t)$ measured at constant $\dot{\gamma}>\dot{\gamma}_{I N}$ as a function of $t_{1} m e$ has been observed and investigated in detail. In agreement with the picture of the first-order phase transition (in the domain of metastability), the transient behavior could be interpreted quantitatively in terms of nucleation and one-dimensional growth process. These results are finally compared to recent predictions by Spenley, Cates and MacLeish (Ref. [7]) who described the nonlınear rheology of wormlike micelles in terms of mechanical instability of shear-banding type.
\end{abstract}

\section{Introduction.}

In spite of an apparent complexity, surfactant solutions of elongated wormlike micelles (also termed equilibrium polymers) exhibit a very simple linear viscoelasticity. In the semidilute

( ${ }^{+}$U.R.A. 233. 
regime of entangled network, the linear rheology is dominated by reptation (as for ordinary polymers) and by reversible breaking and recombination of the chains. The two relaxation times $\tau_{\text {rept }}$ and $\tau_{b}$ related to the previous mechanisms control the dynamical response to an external applied shear. As far as the breaking time $\tau_{b}$ is much shorter than the reptation one. the linear response function $G(t)$ measured in stress relaxation experiments follows an almost pure Maxwell behavior: $G(t)=G_{0} \exp \left(-t / \tau_{\mathrm{R}}\right)$ where $G_{0}$ is the plateau modulus and $\tau_{\mathrm{R}}$ the terminal relaxation time. This Maxwell behavior has been observed on a large variety of materials forming equilibrium polymers [1-6].

In the present paper, we give a comprehensive account for an investigation of the nonlinear rheology of wormlike micellar solutions, which, in contrast to the linear regime, is much less understood.

Our first motivation was a recent theoretical work by Spenley, Cates and McLeish [7] of the nonlinear viscoelastic behavior of wormlike micelles submitted to a steady shear in the semidilute regime. The predictions of these authors (referred to as SCM theory in the following) are based on a so-called reptation-reaction model developed previously by Cates [8] and solved in reference [7] using the constitutive equations of the polymer dynamics [9] adapted to reversibly breakable chains. The main results of this model are the following : as shear reaches a threshold value $\dot{\gamma}_{1}=2.6 / \tau_{\mathrm{R}}$, a mechanical instability of shear-banding type occurs within the micellar solutions. This instability is characterized above $\dot{\gamma}_{1}$ by a plateau of the shear stress $\sigma$ at the height $\sigma^{*}=2 G_{0} / 3$. The plateau persists over a finite $\dot{\gamma}$-range, $\dot{\gamma}_{1}<\dot{\gamma}<\dot{\gamma}_{2}$. Above $\dot{\gamma}_{2}$, the shear stress $\sigma(\dot{\gamma})$ starts to increase again linearly with respect to $\dot{\gamma}$. Actually, the existence of a plateau in the $\sigma(\dot{\gamma})$ behavior at a level $\sim 0.6 G_{0}$ has been reported experimentally, e.g. in $\mathrm{CPCl} / \mathrm{Sal}$ solutions [2] (at low concentrations and without salt added) and for CTAB surfactant solutions at high salt $(\mathrm{KBr})$ contents [3].

The major requirement in order to test the predictions of reference [7] is thus to find out a system of equilibrium polymers whose linear rheological response is as close as possible to that of a Maxwell relaxator. The surfactant solutions made of cationic surfactants (cetylpyridinium ions) diluted in brine $(0.5 \mathrm{M} \mathrm{NaCl})$ with strongly binding counterions (salicylate ions) seem to correspond to the above requirements. In our previous work [6], the linear rheological properties have been studied for concentrations $\phi$ ranging between 1 and $10 \%$. We have shown that the viscoelastic quantities $\eta_{0}, G_{0}$ and $\tau_{\mathrm{R}}$ scale with the surfactant concentrations, with exponents 3.3, 2.2 and 1.1 respectively. These values are in agreement with the exponents expected from a recent stress relaxation model (3.5, 9/4 and 5/4) [10]. Moreover, we were able to deduce the total average length of micellar aggregates $\bar{L}$ from high frequency data of the complex elastic modulus. Contrary to earlier reports $[3,11], \bar{L}$ was found to increase as $\bar{L} \sim \phi^{03-04}$. again very close to the $\phi^{05}$-behavior obtained from simple thermodynamic considerations. For these reasons, we believe that the $\mathrm{CPCl}+\mathrm{Sal}$ micellar system is a very good candidate to test the model of Spenley et al. with respect to the nonlinear rheological predictions.

Our second motivation is based on very recent results on an isotropic-to-nematic transition induced by shear. The first experimental evidence of such a transition has been obtained by Schmitt et al. [12] from the system $\mathrm{CPClO}_{3} / \mathrm{NaClO}_{3} / \mathrm{H}_{2} \mathrm{O}$. We have also observed the unambiguous signature of this transition in the micellar solution $\mathrm{CPCl} / \mathrm{Hex}$ anol/brine [13]. The isotropic-to-nematic $(\mathrm{I} / \mathrm{N})$ transition has been established in both cases from small-angle neutron scattering (SANS) under shear and nonlinear rheology. The features of the transition are the following : i) beyond a transition shear rate, which we hereafter denote $\dot{\gamma}_{\mathrm{I} / \mathrm{N}}$, crescentlike scattering patterns characteristic of orientational nematic order show up in the direction perpendicular to the flow. These peaks coexist over a rather broad $\dot{\gamma}$-range with the ring-like 
pattern of the remaining isotropic phase. ii) At $\dot{\gamma}_{1 / N}$, an abrupt change of slope in the $\sigma(\dot{\gamma})$-behavior is observed. For these two systems, however, the shear-induced transition is observed only at high surfactant concentrations $(\phi>25 \%)$. At such $\phi$, the micelles are quite closely packed, as shown by the correlation bump present in the scattering structure factor. We therefore expect that some prenematic short-range order preexists in the isotropic concentrated solutions and that moderate shearing should easily trigger the $\mathrm{I} / \mathrm{N}$ transition.

In the present paper, we report on the linear and nonlinear rheology of $\mathrm{CPCl} / \mathrm{Sal}$ micellar solutions for total surfactant concentrations $\phi$ ranging from $1 \%$ to $30 \%\left(T=25{ }^{\circ} \mathrm{C}\right)$. We thus investigate the semidilute and the concentrated regimes (the crossover between the dilute and semidilute ranges was estimated to be $\left.\phi^{*}=0.3 \%[6]\right)$. We are then able to check the theoretical predictions of reference [7] and to compare our data to the experimental findings reported on the $\mathrm{I} / \mathrm{N}$ transition $[12,13]$. In addition, we provide small-angle neutron scattering spectra at rest $\left(\dot{\gamma}=0 \mathrm{~s}^{-1}\right)$ on deuterated solutions in order to follow the evolution of local correlations throughout the isotropic range. The main result of this study is the evidence of the shear-induced $\mathrm{I} / \mathrm{N}$ transition down to surprisingly low micellar concentrations (at least down to $\phi \approx 6 \%$ ). In the concentrated regime, the transition is strongly first-order. But the first-order character weakens upon increasing dilution, suggesting that at some critical concentration $\phi_{\mathrm{c}}$ it becomes second-order. Below $\phi_{\mathrm{c}}$, the transition ceases to occur : the $\sigma(\dot{\gamma})$-behavior rather indicates a progressive and homogeneous orientation of the micelles throughout the sample. Moreover, in the two-phase domain (where both isotropic $\left(L_{1}\right)$ and nematic $\left(N_{c}\right)$ phases coexist) a transient behavior of the shear stress $\sigma(t)$ has been observed and investigated in detail. In agreement with the picture of the first-order phase transition, the transient behavior could be interpreted quantitatively in terms of nucleation and one-dimensional growth process of the nematic phase within the isotropic one.

\section{Experimental details and results.}

2.1 EXPERIMENTAL DETAILS. - The surfactant solutions investigated here are the binary mixtures made of cetylpyridinium chloride $\left(\mathrm{CP}^{+}, \mathrm{Cl}^{-}\right)$and sodium salicylate $\left(\mathrm{Na}^{+}, \mathrm{Sal}^{-}\right)$ (hereafter abbreviated as $\mathrm{CPCl} / \mathrm{Sal}$ ) diluted in $0.5 \mathrm{M} \mathrm{NaCl}$-brine. Extensively studied by Hoffmann and coworkers [2] but essentially without added salt, this system is known to easily form elongated wormlike micelles. Our choice of a rather concentrated brine solution was dictated by the requirement of a constant electrostatic screening while varying the surfactant concentrations.

The phase diagram of $\mathrm{CPCl} / \mathrm{Sal}$ solutions is displayed in figure 1 for $\mathrm{CPCl}$ and Sal concentrations $\phi_{\mathrm{CPCl}}$ and $\phi_{\mathrm{Sal}}$ up to $40 \%$ and $12 \%$, respectively $\left(T=30^{\circ} \mathrm{C}\right.$ ). It was derived using $\mathrm{X}$-ray and birefringence measurements. Beyond a very broad isotropic range, a nematic calamitic $\left(\mathrm{N}_{\mathrm{c}}\right)$ «island $»$ is observed for total surfactant concentration $\phi=\phi_{\mathrm{CPCl}}+\phi_{\mathrm{Sal}} \sim 36 \%$, followed at much higher $\phi$ by a hexagonal $(\mathrm{H})$ phase. The sequence $\mathrm{L}_{1} / \mathrm{N}_{\mathrm{c}} / \mathrm{H}$, as well as the typical shape of the $\left(\phi_{\mathrm{CPCl}}, \phi_{\text {Sal }}\right)$-phase diagram resembles strongly that obtained for the $\mathrm{CPCl} / \mathrm{Hexanol} / \mathrm{H}_{2} \mathrm{O}$ micellar solutions $[13,14]$. For the present study, the samples were prepared on the dilution line at constant molar ratio $R=[\mathrm{Sal}] /[\mathrm{CPCl}]=0.5(\phi<30 \%)$.

The linear and nonlinear viscoelastic properties of the $\mathrm{CPCl} / \mathrm{Sal}$ solutions were obtained on a Rheometrics Fluid Spectrometer (RFS II) working in a cone-and-plate configuration with controlled shear rate. Dynamical measurements were carried out for angular frequency $\omega=0.1-100$ rad. $\mathrm{s}^{-1}$ at temperature $T=25^{\circ} \mathrm{C}$. Steady shear rate measurements were restricted to $\dot{\gamma}$ not larger than $100 \mathrm{~s}^{-1}$, because of mechanical flow instabilities such as elasticallydriven meniscus distorsions [15]. We also present small-angle neutron scattering measurements performed at the Orphée Reactor (PAXY instrument) at the Laboratoire Léon Brillouin on 


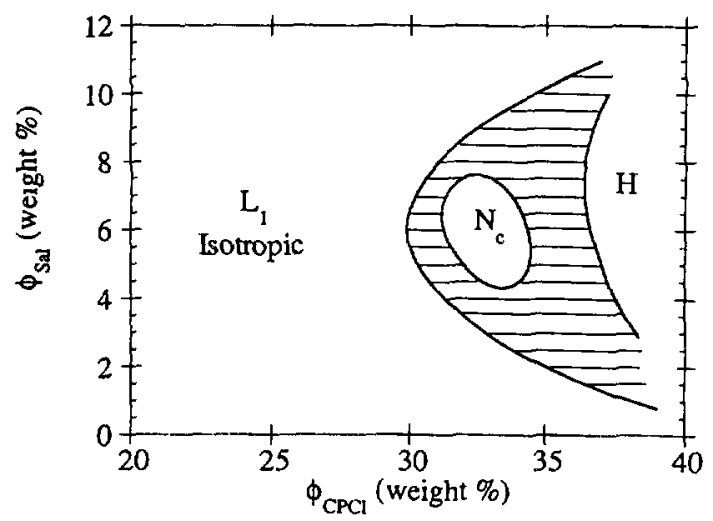

Fig. 1. - Static phase diagram of the ternary surfactant system cetylpyridinium chloride/sodium salicylate $/ 0.5 \mathrm{M} \mathrm{NaCl}$ brine (at $T=30^{\circ} \mathrm{C}$ ) showing the structural sequence isotropic $\left(\mathrm{L}_{1}\right.$ )-nematic calamitic $\left(N_{c}\right)$-hexagonal $(H)$. The long-range orientational order of the different mesophases is related to the orientational degrees of freedom of the micellar aggregates. The hatched area denotes a multiphasic coexistence region. Note that the limits are only indicative and the $\mathrm{CPCl}$ - and Sal-concentrations are given in weight percent.

isotropic $\mathrm{CPCl} / \mathrm{Sal} / \mathrm{D}_{2} \mathrm{O}(0.5 \mathrm{M} \mathrm{NaCl})$ solutions at $\phi=7 \%, 10 \%$ and $25 \%$. The neutron data have been collected on a two-dimensional detector located at $3 \mathrm{~m}$ with a neutron wavelength $\lambda=6.29 \AA$. They have been corrected using the standard procedures developed at the Laboratoire Léon Brillouin.

2.2 SMALL-ANGLE NEUTRON SCATTERING AT REST $\left(\dot{\gamma}=0 \mathrm{~s}^{-1}\right)$. - In figure 2 are displayed the SANS scattering patterns obtained in the $Q$-range $\left[0.01 \AA^{-1}-0.12 \AA^{-1}\right]$ for the $\mathrm{CPCl} / \mathrm{Sal}$

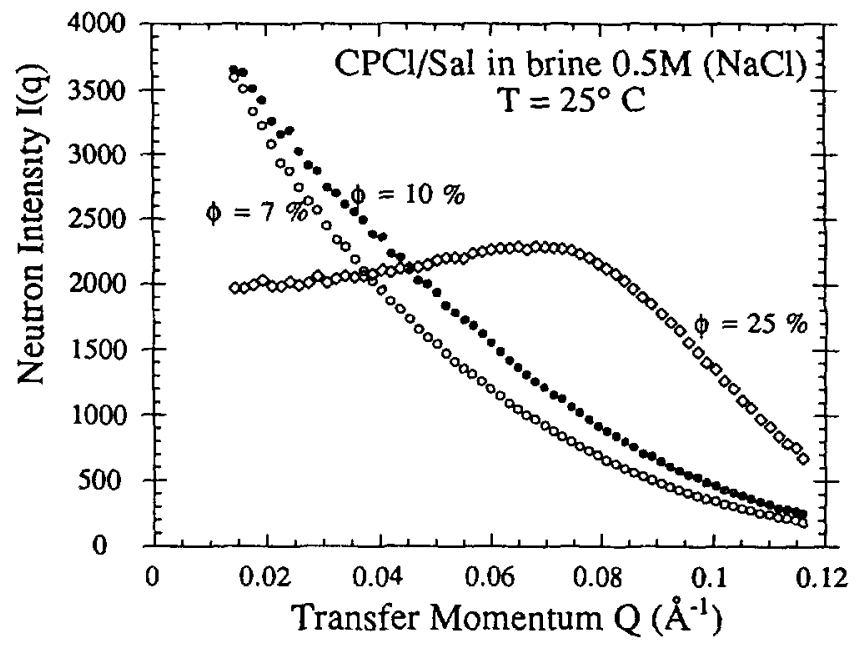

Fig. 2. - Neutron intensity scattered from $\mathrm{CPCl} / \mathrm{Sal}$-solutions of elongated wormlike micelles in the semidilute $(\phi=7$ and $10 \%)$ and concentrated $(\phi=25 \%)$ regimes. The bump showing up at $Q_{\mathrm{m}}=0.07 \AA^{-1}$ for the $25 \%$-sample indicates translational short-range order of the cylindrical aggregates (mean distance $\sim 100 \AA$ ). 
system diluted in deuterated water. The concentrations investigated are $\phi=7,10$ and $25 \%$. At the lowest concentration, the scattering structure factor decreases monotonically with increasing wave-vector, as expected for semidilute solutions of entangled cylindrical aggregates [16]. Analyzing the neutron intensity in terms of $\log [Q \times I(Q)]$ versus $Q^{2}$ plots, we obtain in the high $Q$-region a straight line whose slope is related to the radius $R_{\mathrm{c}}$ of the aggregates (namely $R_{\mathrm{c}}^{2} / 4$ in case of cylinders) [17]. From the two sets of data presented in figure 2 , we derived a mean radius $R_{\mathrm{c}}=21 \AA$ at $\phi=7 \%$ and $22 \AA$ at $\phi=10 \%$. These values are in very good agrement with those obtained by Hoffman et al. [18] on the same surfactant solutions without added salt. On the contrary, for the $25 \%$-solution, a broad correlation bump is observed around $Q_{\mathrm{m}}=0.07 \AA^{-1}$ (mean distance $\sim 100 \AA$ ). The $Q_{\mathrm{m}}$-value coincides approximately with the first Bragg maximum expected for a hexagonal phase made of wormlike micelles at the same concentration. It is therefore not surprising that in concentrated but isotropic solutions, orientational short-range order of the nematic kind preexists and plays an important role.

2.3 LINEAR RHEOLOGY. - A detailed account of the linear viscoelastic properties of CPCl/Sal solutions for $\phi \leqslant 10 \%$ has already been provided in reference [6]. Here we only extend our previous measurements in the concentrated regime, up to $\phi \sim 30 \%$. The frequency dependence of the elastic modulus $G^{*}(\omega)=G^{\prime}(\omega)+i G^{\prime \prime}(\omega)$ is displayed in figure 3 using the usual Cole-Cole representation. For $\phi=6 \%$ to $31 \%$, the imaginary part $G^{\prime \prime}(\omega)$ is plotted versus the real part $G^{\prime}(\omega)$ of the viscoelastic linear response. Except for high concentrations, all the $G^{\prime \prime}\left(G^{\prime}\right)$ data sets can be fitted accurately by a semicircle of radius $G_{0} / 2$ (as shown by the continuous lines), according to :

$$
\left(G^{\prime}-G_{0} / 2\right)^{2}+G^{\prime 2}=G_{0}^{2} / 4 .
$$

This viscoelastic behavior is that of a Maxwell element [19] characterized by a unique relaxation time $\tau_{\mathrm{R}}$. The viscoelastic parameters $G_{0}, \tau_{\mathrm{R}}$ and $\eta_{0}=G_{0} \tau_{\mathrm{R}}=\lim \left|G^{*}(\omega)\right| / \omega$ as

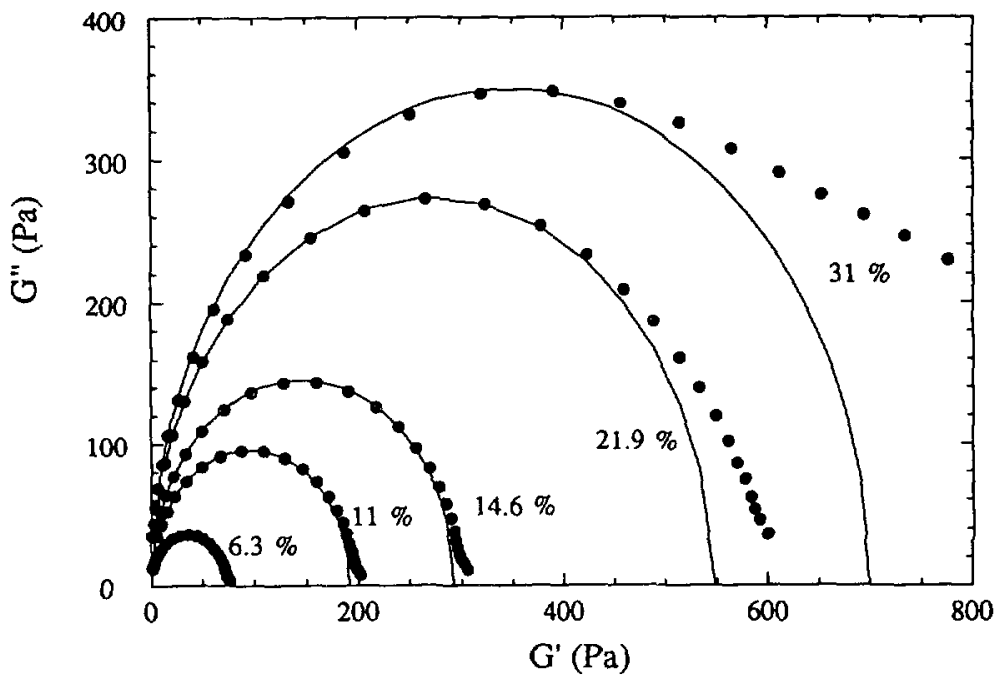

Fig. 3. - Cole-Cole plots for $\mathrm{CPCl} / \mathrm{Sal}$ micellar solutions at various surfactant concentrations $\phi=6-31 \%\left(T=25^{\circ} \mathrm{C}\right)$. Full lines are the results of best fit calculations using equation (1) and correspond to an ideal Maxwell element with a unique relaxation time. 
$\omega \rightarrow 0$ are then easily derived and their $\phi$-dependence is shown in figure 4 . As emphasized in reference [6], the three quantities scale with the surfactant concentration in the semidilute range $(0.4 \leqslant \phi \leqslant 6 \%)$ with exponents in agreement with theoretical predictions [10]. Beyond this range, deviations from the predicted power laws occur. However, the most noticeable features of the concentrated regime are the maxima exhibited by the terminal relaxation time $\tau_{\mathrm{R}}$ around $\phi \sim 10 \%$ and by the static viscosity around $\phi \sim 25 \%$.

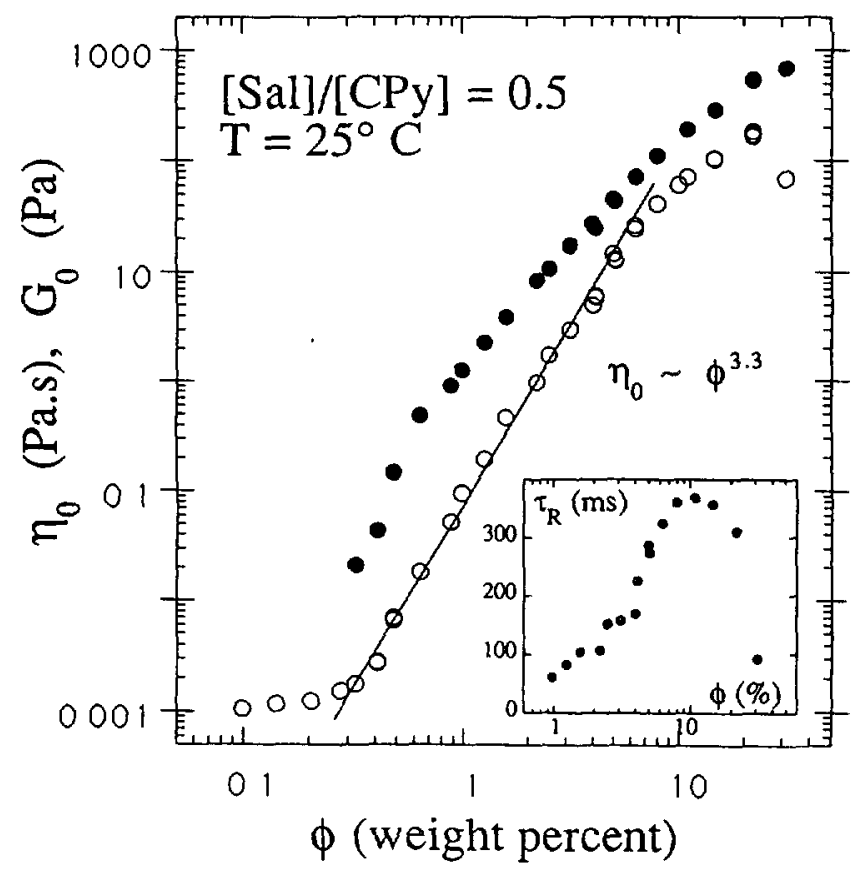

Fig. 4. - Zero-shear viscosity $\eta_{0}$ (empty circles) and elastic plateau modulus $G_{0}$ (full circles) as a function of the surfactant concentration $\phi$ for $\mathrm{CPCl} / \mathrm{Sal}$ solutions of equilibrium polymers. In the semidilute regime, $\eta_{0}$ scales with $\phi$ as $\sim \phi^{3.3}$ (see straight line). Inset : variation of the terminal relaxation tıme $\tau_{R}=\eta_{0} / G_{0}$ against concentration. Note that in the concentrated regime, both $\eta_{0^{-}}$and $\tau_{\mathrm{R}}$-data pass through a maximum.

2.4 NONLINEAR RHEOLOGY. - The nonlinear response of the $\mathrm{CPCl} / \mathrm{Sal}$ surfactant solutions $(R=0.5)$ to steady shear flow is shown in figure 5 for several concentrations. Plotted on a double logarithmic scale, the shear stress $\sigma(\dot{\gamma})$ exhibits an overall analogous behavior for $\phi=5 \%-21 \%$. At low $\dot{\gamma}, \sigma$ increases linearily, reaches a $\dot{\gamma}$-independent plateau (of height $\left.\sigma_{\mathrm{I} / \mathrm{N}}\right)$ at the characteristic rate $\dot{\gamma}_{\mathrm{I} / \mathrm{N}}$ and then, at much higher rates increases again. Note that $\sigma_{\mathrm{I} / \mathrm{N}}$ increases with $\phi$, whereas $\dot{\gamma}_{\mathrm{I} / \mathrm{N}}$, the shear rate at which the plateau sets in, is lowered. However, a close inspection of the $\sigma(\dot{\gamma})$-data shows, two types of behavior. Above $8 \%$, the plateau sets up abruptly and a true discontinuity of slope occurs in the $\sigma(\dot{\gamma})$-behavior at $\dot{\gamma}_{1 / N}$. For $\phi<8 \%$ the transition range between the Newtonian and the plateau regimes is much smoother and clearly rounded. For the $5 \%$-sample, the plateau region has completely disappeared : the only reminiscence of it is an inflexion point occurring in the intermediate $\dot{\gamma}$-range. It should be emphasized finally that the data points of figure 5 have been checked to 


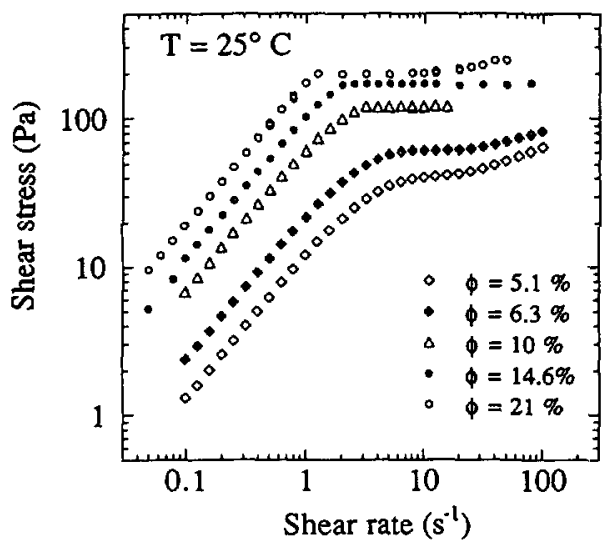

Fig. 5. - Shear rate dependences of the shear stress $\sigma$ measured from $\mathrm{CPCl} / \mathrm{Sal}$ micellar solutions for concentrations $\phi=5 \%-21 \%$. All data points in the plateau regime (see text) were obtained in the stationary limit, i.e. after an equilibration time larger than a few hundred seconds.

be the stationary values of the shear stress, that is, after equilibration times typically longer than $500 \mathrm{~s}$ (see next section).

The dynamical and the steady shear viscosities $\eta(\omega)$ and $\eta(\dot{\gamma})=\sigma / \dot{\gamma}$ are compared to each other in figures $6 \mathrm{a}$ and $6 \mathrm{~b}$ for the $\phi=14 \%$ and $\phi=21 \%$ samples, respectively. Indeed, there is no general reason why dynamic and steady shear data should be identical in any system, as one data set is obtained from linear measurements whereas the other one is taken in highly nonlinear conditions. However, for many systems such as solutions of polymers [19], it happens that both dynamic and steady shear viscosities behave similarily as a function of their respective arguments. This is known as the Cox and Merz empirical rule. In this context, it is worth mentioning that in the case of $\mathrm{CPCl} / \mathrm{Sal}$ equilibrium polymers, dynamic and steady shear viscosities strongly deviate from each other, namely for $\dot{\gamma}>\dot{\gamma}_{\mathrm{I} / \mathrm{N}}$.

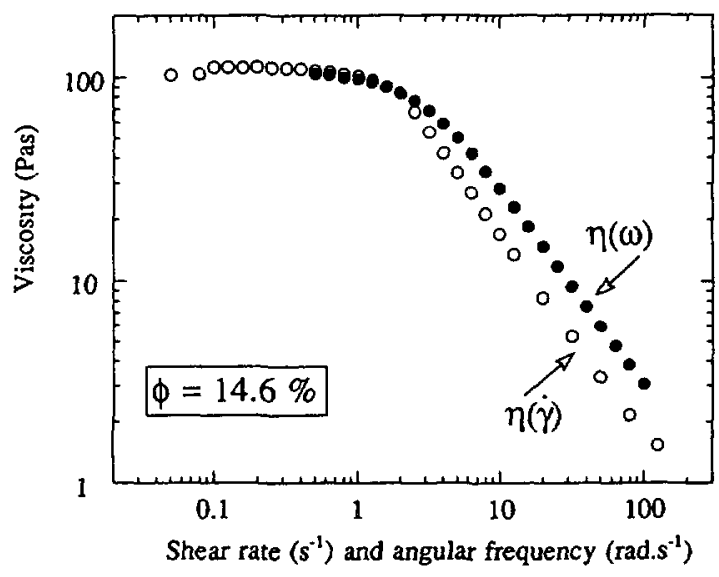

a)

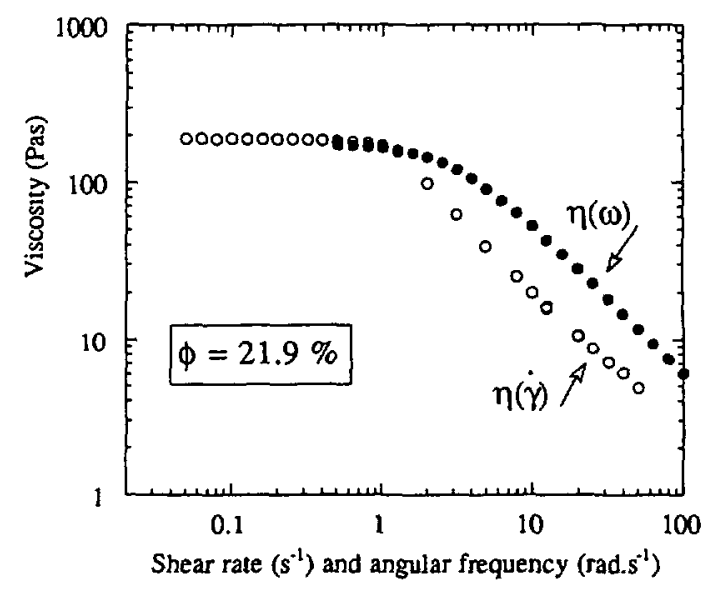

b)

Fig. 6. - Comparison between the dynamical and the steady shear viscosities, $\eta(\omega)$ and $\eta(\dot{\gamma})$ respectively plotted against $\omega$ and $\dot{\gamma}$, for the $\phi=14 \%$ (a) and $\phi=21 \%$ (b) solutions. Deviations of $\eta(\dot{\gamma})$ with respect to $\eta(\omega)$ are observed precisely at the isotropic-to-nematic transition, at $\dot{\gamma}=\dot{\gamma}_{1 / N}$. 
2.5 TRANSIENT BehaVior of THE SHEAR STRESS IN THE PLATEAU REgime. - In the previous section, it has been mentioned that the $\sigma(\dot{\gamma})$-data of figure 5 were obtained in the stationary limit, i.e. after very long equilibration times. Actually, for $\phi>8 \%$ and in the plateau region solely $\left(\sigma=\sigma_{1 / N}\right)$, the shear stress $\sigma$ as received from constant shear rate measurements exhibits a transient behavior. The experiment is performed as follows : firstly the sample is left still to equilibrate at rest in the cone-and-plate apparatus. Shear is then abruptly switched on at a constant value $\dot{\gamma}$ and the stress response $\sigma(t)$ is recorded over time. Typical $\sigma(t)$-variations are illustrated in figures $7 \mathrm{a}$ and $7 \mathrm{~b}$ for the $14 \%$-sample for $\dot{\gamma}=1-5 \mathrm{~s}^{-1}$ Below $\dot{\gamma}_{\mathrm{I} / \mathrm{N}}$, the stationary limit is reached very rapidly, namely within $\tau_{R}(\leqslant 1 \mathrm{~s})$, as shown by the 1 and $2 \mathrm{~s}^{-1}$-data in figure 7a. For shear rates exceeding $\dot{\gamma}_{\mathrm{I} / \mathrm{N}}$, an overshoot is clearly identified. As soon as the shearing is switched on, $\sigma(t)$ first increases rapidly up to a value denoted $\sigma(0)$ (the rising times remains below $1 \mathrm{~s}$ ), and then the stress relaxes slowly down to its stationary limit $\sigma_{1 / N}$. Typical time scales for this kinetics are $10 \mathrm{~s}-200 \mathrm{~s}$, depending on the shear rate applied. It must also be noted in figure $7 \mathrm{~b}$ that at $\dot{\gamma}=5 \mathrm{~s}^{-1}$ not only do we observe an overshoot, but it is followed by a small undershoot. This feature is presently not understood. However, it has been carefully checked that this undershoot is not observed as long as the excess shear rate $\dot{\gamma}-\dot{\gamma}_{\mathrm{I} / \mathrm{N}}$ is moderate (corresponding to $\sim\left(\sigma(0)-\sigma_{\mathrm{I} / \mathrm{N}}\right) / \sigma_{\mathrm{I} / \mathrm{N}}<50 \%$ ).

This transient behavior deserves further comments. The same stationary limit $\sigma_{\mathrm{I} / \mathrm{N}}=170 \mathrm{~Pa}$ is obtained for the 3,4 and $5 \mathrm{~s}^{-1}$ data sets, in agreement with the steady state measurements of figure 5. Moreover, the higher the shear rate applied onto the sample $\left(\dot{\gamma}>\dot{\gamma}_{\mathrm{IN}} \approx 2 \mathrm{~s}^{-1}\right)$, the faster the relaxation. As a consequence of the strong variation of the relaxation time with $\dot{\gamma}$, the $\sigma(t)$-profiles could be determined properly only in a restricted $\dot{\gamma}$-range, typically a few $\mathrm{s}^{-1}$ above $\dot{\gamma}_{\mathrm{I} / \mathrm{N}}$. It should be noticed that the transient behavior shown for $\phi=14 \%$ in figure 7 is representative for the other samples $(\phi=8 \%-31 \%)$ which exhibit similar stress relaxations in the plateau region.

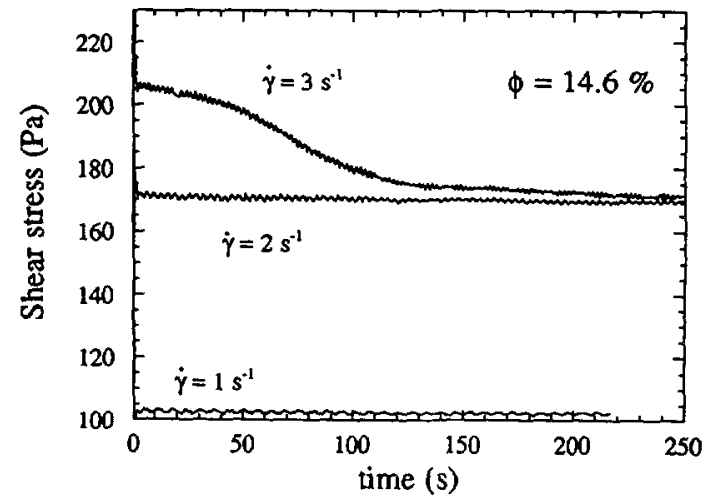

a)

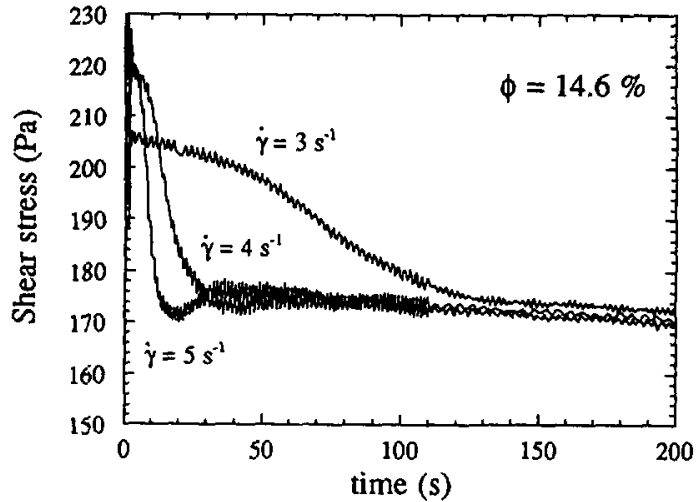

b)

Fig. 7. - Typical transient behavior of the shear stress $\sigma(t)$ in the plateau regime, as received from constant shear rate measurements $\left(\dot{\gamma}=1\right.$ to $\left.5 \mathrm{~s}^{-1}\right)$. The data shown are for $\phi=14.6 \%$. The transition rate is estimated at $\dot{\gamma}_{\mathrm{I} / \mathrm{N}}=2 \mathrm{~s}^{-1}$ and the stationary limit $\sigma_{\mathrm{I} / \mathrm{N}}=170 \mathrm{~Pa}$.

\section{Analysis and discussion.}

The main point that we will attempt to establish in this section is that the results reported above can be interpreted in terms of first-order phase transition induced by shear, from an isotropic to a nematic phase. 
3. 1 THE Linear Regime. - Before doing so, we first discuss briefly the linear viscoelastic properties of the $\mathrm{CPCl} / \mathrm{Sal}$ equilibrium polymers shown in figures 3 and 4 . As emphasized in reference [6], the theoretical predictions derived for solutions of long flexible micelles apply fairly well over an exceptionally large concentration range (at given temperatures and salt contents). A Maxwell-type relaxation behavior of the linear response function is observed for $\phi \approx 2 \%-20 \%$. This implies that the low-frequency elastic modulus $G^{*}(\omega)$ (with $\omega<100 \mathrm{rad} . \mathrm{s}^{-1}$ ) is entirely described through two parameters, $G_{0}$ and $\tau_{\mathrm{R}}$. Moreover, in the range where the Cole-Cole plots are semicircular, we can assume, following Cates [10], that the time for reversible scission of the aggregates is always much lower than that of reptation : $\tau_{\mathrm{b}} \ll \tau_{\text {repl }}$.

The observation of the expected scaling laws for $G_{0}(\phi)$ and $\eta_{0}(\phi)$ with the right exponents (2.2 and 3.3, respectively) in the semidilute regime $(\phi \approx 0.6-6 \%)$ supports the conclusion that the $\mathrm{CPCl} / \mathrm{Sal}$ solutions are a model system for the study of the rheological properties of equilibrium polymers. The deviations of $G_{0}(\phi)$ and $\eta_{0}(\phi)$ from these scaling laws observed in figure 4 at about $6-10 \%$ are probably due to the fact that the system is now in an intermediate regime, in between the pure semidilute $(\sim 1 \%)$ and the concentrated $(\sim 30 \%)$ regimes. Another indication is the direct measurements of the correlation length $\xi$ obtained by using elastic light scattering experiments $[6]:$ at $6 \%, \xi \approx 150 \AA$, that is a value quite similar to what is currently expected for the persistence length of giant micelles [20].

3.2 THE NONLINEAR REgIME. - The nonlinear viscoelastic response of the CPCl/Sal solutions to a steady shear are now analyzed in terms of dimensionless units : the shear stress $\sigma$ has been normalized at each $\phi$ with respect $G_{0}$ and plotted in figure 8 against the shear rate, itself rescaled in terms of $\dot{\gamma} \tau_{R}$. As already mentioned, the terminal relaxation time $\tau_{R}$ and the elastic

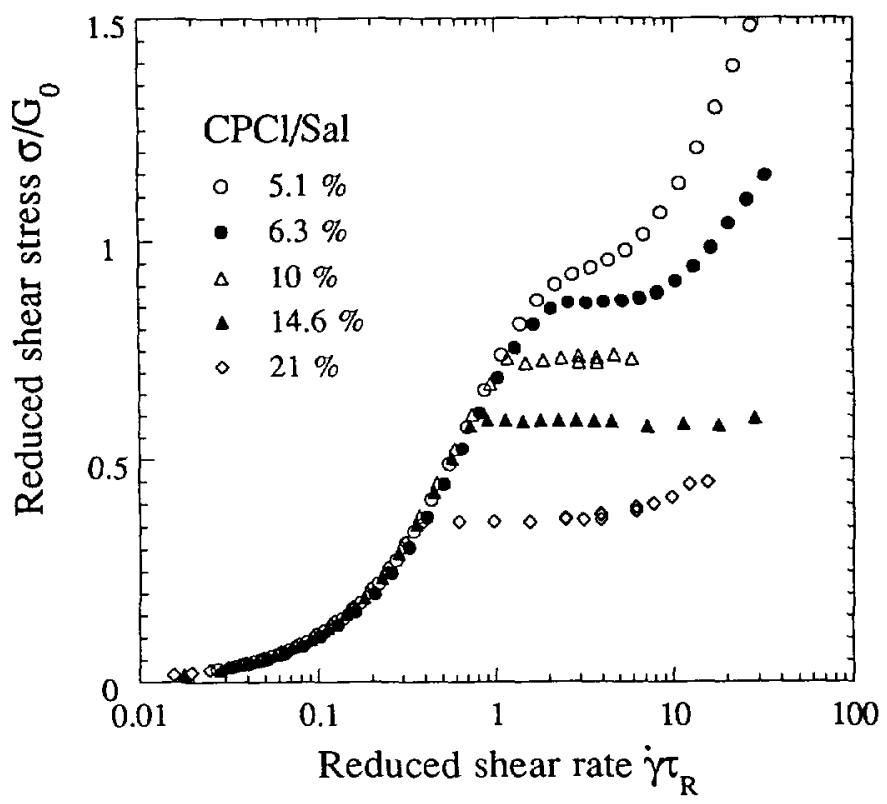

Fig. 8. - Same data as for figure 5 but in dimensionless units. For each $\phi$, the shear stress $\sigma$ has been normalized with respect to the elastic plateau modulus $G_{0}$ and plotted against $\dot{\gamma}$, itself normalized with respect to the inverse relaxation time, $1 / \tau_{\mathrm{R}}$. 
plateau modulus $G_{0}$ are the two parameters entirely describing the linear regime. These two quantities, $\sigma / G_{0}$ and $\dot{\gamma} \tau_{\mathrm{R}}$ are fundamental for the rheology of these systems : $\dot{\gamma} \tau_{\mathrm{R}}$ denotes the Weissenberg number $W_{1}$ (usually defined as the product of the velocity gradient and a characteristic relaxation time [15]) and $\sigma / G_{0}$ can be viewed as the shear stress per structural units of the entangled network (since $G_{0} \sim \xi^{-3}, \xi$ being the size of the blob in the semidilute description [9]).

Moreover, an advantage of using dimensionless units is that the mechanical response of micellar systems at different concentrations can be directly compared. In the low $\dot{\gamma}$-range, as expected from the Newtonian regime, the $\sigma / G_{0}$-data versus $\dot{\gamma} \tau_{\mathrm{R}}$ are found to be superimposed. At higher shear rate, the evolution of the different samples deviates markedly from each other. Now, the plateau sets in at $\dot{\gamma}_{\mathrm{I} / \mathrm{N}} \tau_{\mathrm{R}}$ and at the level $\sigma_{\mathrm{I} / \mathrm{N}} / G_{0}$. both quantities being strongly $\phi-$ dependent. The concentration dependence of the Weissenberg number characterizing the $\mathrm{I} / \mathrm{N}$ transition $\dot{\gamma}_{\mathrm{I} / \mathrm{N}} \tau_{\mathrm{R}}$ and the rescaled stress $\sigma_{\mathrm{I} / \mathrm{N}} / G_{0}$ in the plateau regime are displayed in figure 9 . For $\phi \approx 6 \%, W_{1}$ remains unchanged at $2.7 \pm 0.2$. At higher $\phi$, it decreases as $\sim \phi^{-3 / 2}$ (the straight line in Fig. 9), whereas $\sigma_{1 / \mathrm{N}} / G_{0}$ is decreased by almost a decade on the same concentration range. The last data set will be utilized to determine the dynamical phase diagram of the CPCl/Sal system.

At this point it is worth comparing our results to the predictions of the Spenley, Cates and McLeish (SCM) theory on the nonlinear rheology of wormlike micelles [7]. As pointed out in the introduction, according to the SCM theory, a stress saturation similar to that obtained for the $\mathrm{CPCl} / \mathrm{Sal}$ equilibrium polymers (Fig. 8) is predicted above a characteristic shear rate $\dot{\gamma}_{1}$. But once expressed in reduced units, the mechanical behavior predicted for different concentrations is identical. The corresponding « universal » critical reduced shear rate and stress are such as : $W_{1}=\dot{\gamma}_{1} \tau_{\mathrm{R}}=2.6$ and $\sigma^{*} / G_{0}=2 / 3$. In this respect, the SCM predictions seem to disagree with the observations of figure 9 , where at large $\phi, \dot{\gamma}_{1 / \mathbb{N}} \tau_{\mathrm{R}} \sim \phi^{-3 / 2}$ However, the «critical » parameters $\dot{\gamma}_{1}$ and $\sigma^{*}$ in the SCM theory are derived from a criterion of complete mechanical instability : they correspond to a state of shearing where the flow becomes unstable (i.e. related to a situation where $\mathrm{d} \sigma / \mathrm{d} \dot{\gamma}$ becomes negative) and the medium separates into a fluid phase of high shear rate and a viscous phase of low shear rate (the socalled shear-banding instability). Nevertheless the possibility remains that the fluid phase

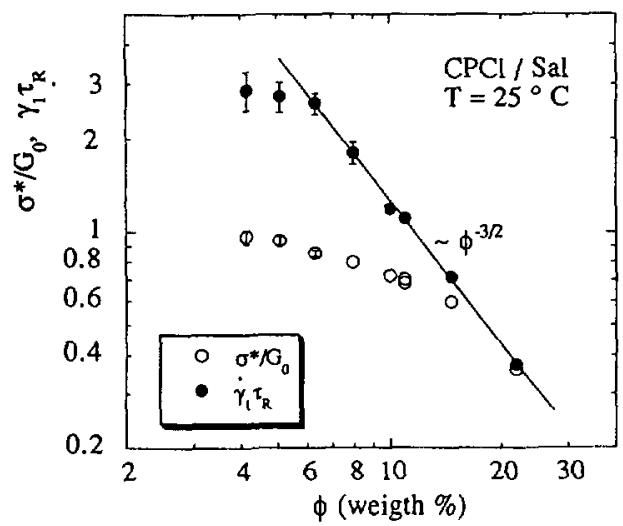

Fig. 9. - Concentration dependence of the rescaled shear stress $\sigma_{1 / N} / G_{0}$ at the plateau and of the Weissenberg number $W_{1}=\dot{\gamma}_{\mathrm{I} / \mathrm{N}} \tau_{\mathrm{R}}$ characterizing the $\mathrm{I} / \mathrm{N}$ transition. As long as the transition is of firstorder type, $W_{1}$ is found to scale with $\phi^{-3 / 2}$ 
nucleates much before the criterion of mechanical instability is reached, corresponding to $\dot{\gamma}_{1 / N}<\dot{\gamma}_{1}$. In this case, the SCM conceptual framework would not be incompatible with the present results.

It is clear from figure 8 that for the most concentrated sample $(\phi=21 \%)$, the plateau sets in very abruptly, and the deviations from the Newtonian regime now occur at very low Weissenberg number $\left(W_{1} \approx 0.2\right)$. As mentioned in the introduction, such an abrupt change of slope in the $\sigma(\dot{\gamma})$-behavior has been encountered in concentrated micellar solutions of $\mathrm{CPClO}_{3} / \mathrm{NaClO}_{3} / \mathrm{H}_{2} \mathrm{O}$ [12] and $\mathrm{CPCl} /$ hexanol/ $\mathrm{H}_{2} \mathrm{O}$ [13]. Only small-angle neutron scattering could show in these systems that the plateau region, for $\dot{\gamma}>\dot{\gamma}_{\mathrm{I} / \mathrm{N}}$ corresponds to a shearing domain where both isotropic and nematic phases coexist. Consistently with the former observations, we interpret the onset of the plateau for the $\mathrm{CPCl} / \mathrm{Sal}$ system as the signature of a shear-induced isotropic-to-nematic phase transition of first-order.

The constancy of the shear stress throughout the plateau can be qualitatively interpreted as follows. In the two-phase domain, the sample is most likely organized in a random succession of macroscopic layers of the $L_{I}$ and $N_{c}$ phases. Due to the orientation of the micelles in the flow direction, the viscosity of the nematic phase is much lower than that of the remaining isotropic phase. At an imposed shear rate, the shear stress is identical through all the sample in both kinds of layers, but the local shear rate is much higher in the fluid nematic layers than in the viscous isotropic ones. Increasing the velocity gradient at the plateau has the effect to increase the total thickness of the nematic layers. Thus only the proportion of one phase with respect to the other changes, and as long as the concentration of each phase is comparable [13], $\sigma(\dot{\gamma})$ remains constant. Due to the instabilities of cone-and-plate type occurring as early as $20 \mathrm{~s}^{-1}$, we could not explore the entire two-phase domain. And then, it is probable that in none of the high concentration data, a fully nematic phase could be induced.

At lower concentrations, the picture changes progressively. For $\phi=6.3 \%$, the onset of the plateau, which now extends over a quite narrow $\dot{\gamma}$-range, is not as abrupt, but somewhat rounded. By continuity with the picture developed previously for higher $\phi$, we keep with the idea of a shear-induced $\mathrm{I} / \mathrm{N}$ transition, but now having a weaker first-order character. On the contrary, at $5 \%$, the plateau is replaced by an inflexion point in the intermediate $\dot{\gamma}$-range. According to this, the picture of the $\mathbf{I} / \mathrm{N}$ transition should not apply for this sample. The deviations from the Newtonian regime are related to a progressive and homogeneous alignment of the polymers through the sample. Extrapolating between the behavior obtained at 5 and $6.3 \%$, there should be a critical concentration $\phi_{c}$ where the otherwise first-order $\mathrm{I} / \mathrm{N}$ transition becomes second-order. This model of first- and second-order phase transition induced by shear is illustrated in figure 10 , which is an attempt to derive a dynamical phase diagram for the $\mathrm{CPCl} / \mathrm{Sal}$ equilibrium polymers. In this figure, the rescaled stress at the plateau $\sigma_{1 / \mathbb{N}} / G_{0}$ is shown versus concentration using linear scales. The straight line through the data points defines the line of coexistence of the $L_{1}$ and $N_{c}$-phases under shear. All along this line the transition is clearly first-order. On one side. it is terminated by a critical point $\left(\mathrm{P}_{\mathrm{c}}\right)$ at $\phi_{c} \approx 6 \%$ where the transition becomes second-order. In the high $\phi$-range, it extrapolates to the biphasic region observed at rest. The biphasic domain in the high concentration and low stress ranges of the phase diagram is not known precisely, and thus the phase boundaries are only indicative in this region.

3.3 NUClEATION AND ONE-DIMENSION GROWTH MODEL. - Nevertheless, from stationary rheological measurements alone, it is rather delicate to demonstrate unambiguously the firstorder character of the transition for $\phi>\phi \ldots$. Fortunately, the transient relaxations observed in the plateau region (Fig. 7) enable us to show the existence of a domain of metastability, which is a fundamental feature of the first-order transition. The metastable region is characterized by a rather slow kinetical evolution [21] (compared to the unstable region inside the spinodal) of 


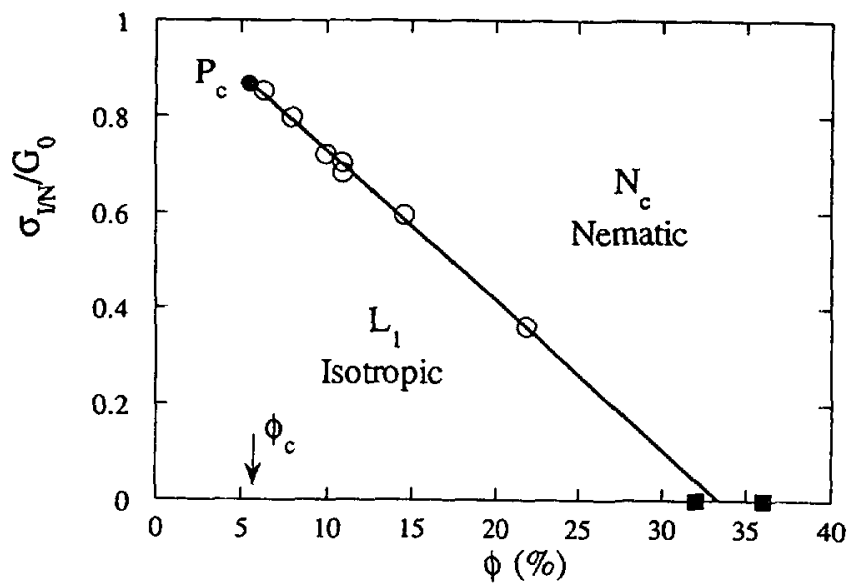

Fig. 10. - Dynamical phase diagram of the CPCl/Sal solutions of equilibrium polymers derived from the nonlinear viscoelastic response of figure 8 . The straight line defines the concentration evolution of the shear stress in the plateau regime, i.e. in the domain of coexistence of the $L_{i}$ and $N_{c}$ phases. All along this line the transition is clearly first-order. On one side, it is terminated by a critical point $\left(P_{c}\right)$ at $\phi_{\mathrm{c}} \approx 6 \%$ where the transition becomes second-order. In the high $\phi$-range, it extrapolates to the biphasic region observed at rest.

the appropriate quantity, here the shear stress. In the case of a system with non conserved order parameter, the kinetics in the domain of metastability is driven by nucleation and growth processes.

We interpret the transient evolution of $\sigma(t)$ as follows. Immediately after the onset of shear at a given $\dot{\gamma}$ (always assuming $\phi>\phi_{\mathrm{c}}$ and $\dot{\gamma}>\dot{\gamma}_{\mathrm{I} / \mathrm{N}}$ ), the solution is still in its disordered state all through the cone-and-plate cell. Due to its higher viscosity, an overshoot is observed and characterized by an amplitude $\Delta \sigma_{0}=\sigma(0)-\sigma_{\mathrm{I} / \mathrm{N}}$. As time evolves, the nematic phase nucleates and grows. Due to its lower viscosity, $\sigma(t)$ monotically decreases towards its final stationary plateau value $\sigma_{1 / N}$. As a first step of our derivation, we search for a relationship between the value of the stress $\sigma(t)$ measured at time $t$ and the volume fraction of the nematic phase already transformed. Let us define $X_{\mathrm{N}}$ as the proportion of nematic phase (relative to the total sample volume) in the stationary limit (i.e. for $t \rightarrow \infty)$ and $X_{\mathrm{N}} X(t)$ the same quantity at time $t$. According to this, $0 \leqslant X(t) \leqslant 1$. Moreover, if we assume that in the two phase-domain the sheared sample exhibits a succession of macroscopic layers of fluid high shear phase $\left(\mathrm{N}_{\mathrm{c}}\right)$ and viscous low shear phase $\left(\mathrm{L}_{1}\right)$, it can be shown that (see Appendix):

$$
\frac{\Delta \sigma(t)}{\sigma(t)}=\frac{\Delta \sigma(0)}{\sigma(0)}[1-X(t)] \text {. }
$$

This relation can be safely linearized, provided that we only investigate situations where the overshoot $\Delta \sigma(0) / \sigma_{1 / N}$ is rather moderate, say below $\sim 50 \%$. Equation (2) then is written :

$$
\Delta \sigma(t) \approx \Delta \sigma(0)[1-X(t)] .
$$

The consequences of this simplifying linearization are further discussed in the Appendix. We now turn to the calculation of the transformed fraction $X(t)$. Since the transition is induced by shear, we expect the growth of the nematic nuclei to be strongly anisotropic. More precisely. we assume that the growth is slower in the direction of the velocity gradient than in 
those of the flow and of the vorticity. So, the rate limiting process of growth essentially acts in one direction (1D) only. Between the time $\tau$ (comprised between 0 and $t$ ) and $\tau+\mathrm{d} \tau$, $N(\tau)\left[1-X_{\mathrm{N}} X(\tau)\right] \mathrm{d} \tau$ denotes the number of nematic nuclei which have nucleated. Here, $N(\tau)$ is the nucleation rate per unit volume and the factor $\left[1-X_{\mathrm{N}} X(\tau)\right]$ accounts for the fact that nucleation can only occur in the volume which has not been yet transformed at this time $\tau$. Thus, during the next interval: $t-\tau$, these nuclei will grow at the rate $C\left(t^{\prime}\right)$, and consequently, the total fraction transformed is written :

$$
X(t)=\int_{0}^{t} N(\tau)\left[1-X_{\mathrm{N}} X(\tau)\right] \mathrm{d} \tau \int_{\tau}^{t} C\left(t^{\prime}\right) \mathrm{d} t^{\prime}
$$

In order to proceed further, we must ascribe the time dependence of the nucleation and growth rates of equation (4), $N(t)$ and $C(t)$. First, it is important to outline that in this kind of phase induced transition, $\Delta \sigma(t)$ plays the role of a driving force. Since the stationary limit is reached at long times $(\Delta \sigma(t \rightarrow \infty)=0)$, the growth must be zero in this limit. The simplest approximation consists in taking $C(t)$ proportional to $\Delta \sigma(t)$ and thus : $C(t)=C_{0} \times \Delta \sigma(t)$. Discussing the time variation of the nucleation rate is less straightforward and thus for the sake of simplicity again $N(t)$ has been considered to be time independent i.e. $N(t)=N_{0}$ (a discussion about the validity of both assumptions is given later on). Moreover, since we are concerned with a restricted $\dot{\gamma}$-region just above $\dot{\gamma}_{I N \mathbb{N}}$, the total fraction transformed $X_{\mathrm{N}}$ is expected to be small compared to unity. Then, we can neglect the product $X_{\mathrm{N}} X(t)$ under the first integral in equation (4). Once differentiated with respect to the time, equation (4) becomes:

$$
\frac{\mathrm{d} X}{\mathrm{~d} t}=C_{0} N_{0} t\left[\sigma(t)-\sigma_{\mathrm{I} / \mathrm{N}}\right]
$$

which, together with equation (3), finally yields a relaxation for the excess stress of the form :

$$
\sigma(t)-\sigma_{\mathrm{I} / \mathrm{N}}=\Delta \sigma_{0} \exp \left(-\frac{t^{2}}{\tau_{\mathrm{M}}^{2}}\right) .
$$

Similar calculations have been performed using a nucleation rate $N(t) \propto \Delta \sigma(t)$. In this case, the result is :

$$
\sigma(t)-\sigma_{\mathrm{I} / \mathrm{N}}=\Delta \sigma_{0}\left[1-\operatorname{th}^{2}\left(\frac{t}{\tau_{\mathrm{M}}}\right)\right] .
$$

In equations (6) and (7), $\tau_{M}$ is the characteristic time of the $\sigma(t)$-evolution, which depends on the unknown numerical prefactors relating the nucleation and growth rates to $\Delta \sigma(t)$. Note that the two expressions given by equations (6) and (7) are asymptotically identical at short times $\left(t \ll \tau_{\mathrm{M}}\right)$, and only deviates from one an other for $t>\tau_{\mathrm{M}}$.

Quantitative comparisons between equations $(6,7)$ and the experimental data are shown in figures $11 \mathrm{a}, \mathrm{b}$ and $\mathrm{c}$ for $\phi=11 \%, 14.6 \%$ and $21 \%$ respectively in terms of the rescaled quantities : $\Delta \sigma(t) / \Delta \sigma_{0}$ versus $t / \tau_{\mathrm{M}}$. One can see that, all data points taken at different $\dot{\gamma}>\dot{\gamma}_{\mathrm{I} / \mathrm{N}}$ are found to collapse on a single master curve. The values of $\sigma_{\mathrm{I} / \mathrm{N}}, \Delta \sigma_{0}$ and $\tau_{\mathrm{M}}$ for the different data sets of figure 11 are given in table 1 . The theoretical curves, which read in these renormalized units $\Delta \sigma(T) / \Delta \sigma_{0}=\exp \left(-T^{2}\right)$ and $\Delta \sigma(T) / \Delta \sigma_{0}=1-\mathrm{th}^{2}(T)$ (see continuous lines in Figs. 11) are in good agreement with the experimental master curve, suggesting that the nucleation and 1D-growth model is indeed appropriate.

Furthermore, other approximations for the time dependence of the rates $N(t)$ and $C(t)$ have been tried and equation (4) solved numerically. These attempts suggest that the 


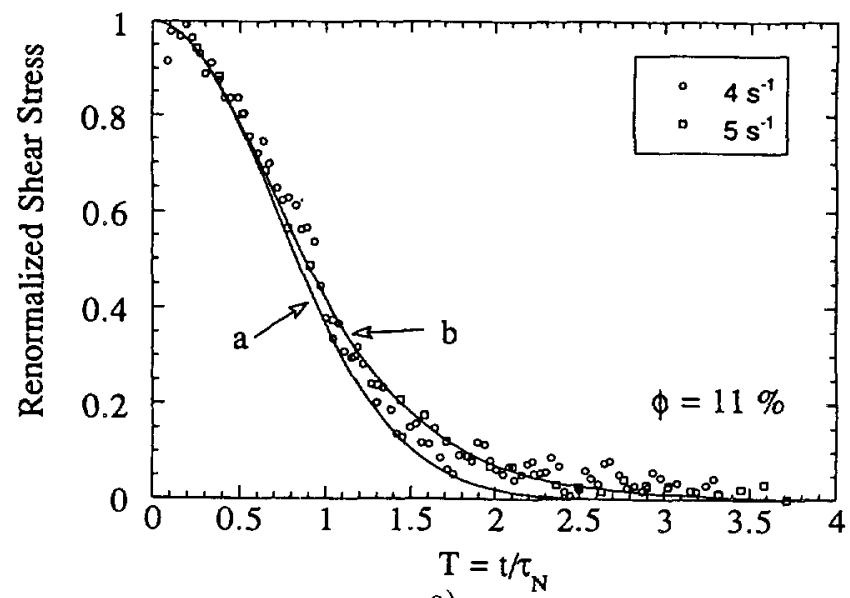

a)

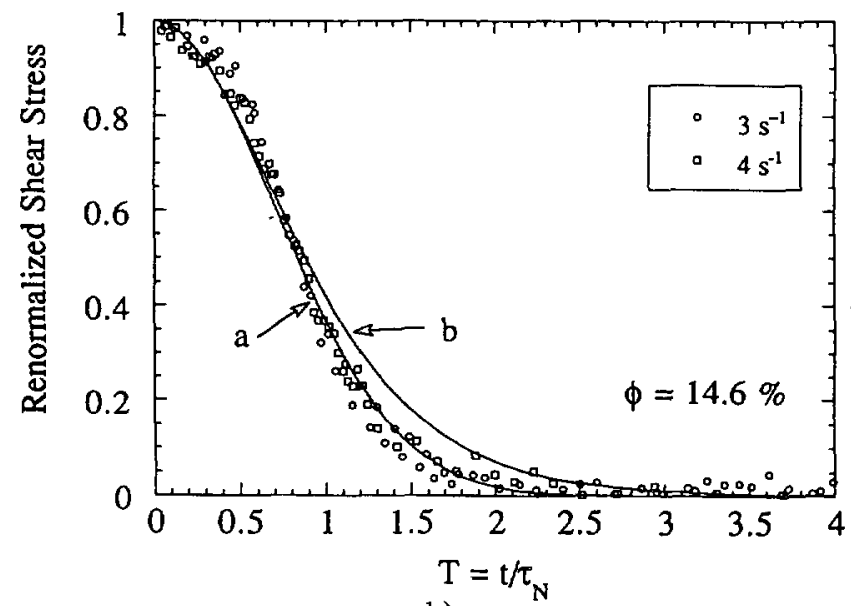

b)

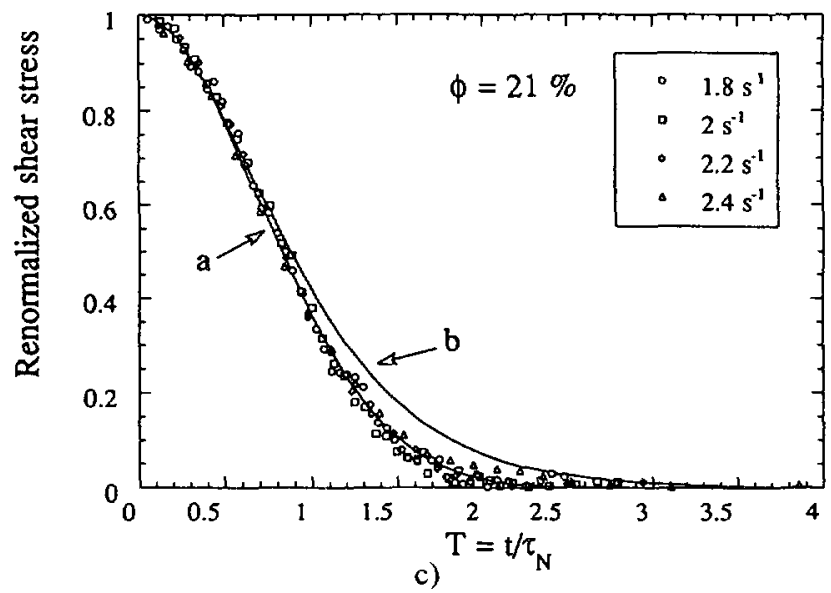

Fig. 11. - Transient behavior obtained in the two-phase domain regime $\left(\dot{\gamma}>\dot{\gamma}_{\mathrm{I} / \mathrm{N}}\right)$ for $\phi=11 \%(\mathrm{a})$, $14.6 \%$ (b) and $21 \%$ (c). The shear stress measured at constant shear rate over time has been normalized with respect to its initial value $\Delta \sigma(0)$. Identically, the time was rescaled with respect to $\tau_{M}$, the nucleation and $1 \mathrm{D}$-growth time of the model. Typical values for the overshoot $\Delta \sigma_{0}$ and $\tau_{M}$ are listed in table I. 
Table I. - Compilation of the parameters $\dot{\gamma}_{\mathrm{I} / \mathrm{N}}, \sigma_{\mathrm{I} / \mathrm{N}}, \Delta \sigma_{0}$ and $\tau_{\mathrm{M}}$ deduced from best fit calculations of the transient evolution of the shear stress $\sigma(t)$ in the nucleation and growth regime ( $\phi>\phi_{\mathrm{c}}$ and $\dot{\gamma}>\dot{\gamma}_{\mathrm{I} / \mathrm{N}}$ ) using equation (6). The values given here have been used to rescale the stress data shown in figures $11 \mathrm{a}, \mathrm{b}$ and $\mathrm{c}$.

\begin{tabular}{|c|c|c|c|c|c|}
\hline & $\dot{\gamma}_{\mathrm{I} / \mathrm{N}}\left(\mathrm{s}^{-1}\right)$ & $\dot{\gamma}\left(\mathrm{s}^{-1}\right)$ & $\sigma_{\mathrm{INN}}(\mathrm{Pa})$ & $\Delta \sigma_{0}(\mathrm{~Pa})$ & $\tau_{\mathrm{M}}(\mathrm{s})$ \\
\hline $\begin{array}{c}\phi=11 \% \\
\text { (cf. Fig. 11a) }\end{array}$ & $\sim 3.5 \mathrm{~s}^{-1}$ & 4.0 & 130 & 7 & 120 \\
\hline$\phi=14.6 \%$ & $\sim 2 \mathrm{~s}^{-1}$ & 3.0 & 131 & 12 & 52 \\
\hline (cf. Fig. 11b) & & 4.0 & 173 & 34 & 87 \\
\hline$\phi=21 \%$ & $\sim 1.5 \mathrm{~s}^{-1}$ & 1.8 & 184 & 33 & 17 \\
(cf. Fig. 11c) & & 2.0 & 185 & 54 & 150 \\
& & 2.2 & 180 & 71 & 52 \\
& & 2.4 & 181 & 81 & 36 \\
\hline
\end{tabular}

relation $C(t) \propto \Delta \sigma(t)$ is a crucial assumption. For instance, a quadratic dependence $C(t) \propto \Delta \sigma(t)^{2}$ would provide a Lorentzian relaxation, which obviously deviates from the master curves. On the other hand, the time variation of the nucleation rate $N(t)$ plays only a minor role on the overall $\sigma(t)$-behavior.

At this point it must be emphasized that the quantitative model of nucleation and $1 \mathrm{D}$-growth reported previously relies on many assumptions. For the growth rate $C(t)$, a linear $\Delta \sigma(t)$-variation was considered whereas the nucleation rate $N(t)$ was taken to be either constant or again linear in $\Delta \sigma(t)$. These relationships are realistic at low excess shear rates $\dot{\gamma}-\dot{\gamma}_{\mathrm{I} / \mathrm{N}}$, only. In particular, they will certainly not apply when approaching the spinodal regime which is expected to take place in the picture of the first order phase transition at much higher shear rates. No matter how we specify the model quantitatively, there remains an experimental fact that seems difficult to interpret even qualitatively. Namely the undershoot displayed in figure $7 \mathrm{~b}$ for the $14.6 \%$ sample at $\dot{\gamma}=5 \mathrm{~s}^{-1}$ Experiments (not shown here) performed at still larger shear rates even showed a damped oscillation behavior for $\Delta \sigma(t)$ toward its stationary value. Such a behavior implies the existence of some «inertial » term in the time differential equation of the stress kinetic : a feature that is not contained in the present description. Clearly a more refined interpretation is still to be built up in order to understand the regime of high excess shear rates.

\section{Concluding remarks.}

When submitted to (high enough) steady shear, the $\mathrm{CPCl} / \mathrm{Sal}$ solutions of equilibrium polymers exhibit a first-order isotropic-to-nematic transition for all surfactant concentrations $\phi>6 \%$. According to the present rheological investigation, the features of the transition are the existence of a true plateau in the $\sigma(\dot{\gamma})$-dependence in the stationary limit and a transient behavior of the shear stress in the same regime. The $\sigma(t)$ transient profiles have been well accounted for by a simple nucleation and 1D-growth model (domain of metastability). Compared to the other systems $[12,13]$ which have been reported to display the I/N transition, the $\mathrm{CPCl} / \mathrm{Sal}$ system presents specific features :

i) the transition persists at surprisingly low concentrations $(\phi \approx 6 \%)$, where the scattering structure factor of the solution (at rest) displays no correlation peak; 
ii) at low concentrations the first-order character of the transition weakens progressively and eventually ends up at a critical concentration $\phi_{\mathrm{c}}$ where it becomes second-order. Below $\phi_{c}$, the transition is no longer observed, but replaced by a progressive and homogeneous orientation of the micellar objects submitted to shear;

iii) below $\phi_{\mathrm{c}}$ we do not observe the mechanical instability predicted by Spenley et al. [7] and experimentally found out in the same $\mathrm{CPCl} / \mathrm{Sal}$ system at lower concentrations and no added salt [2].

In the previous reports on the $\mathrm{I} / \mathrm{N}$ transition [12, 13], it was argued that the short-range prenematic order resulting from the close packing of the micelles at high concentrations plays a crucial role in the onset of the transition. Coupled to the stress, this local order becomes longrange as far as shearing is applied to the solution. In this context, the question arises from point i) is the prenematic order a necessary condition for the transition to occur under shear ? And if it is, how can we rationalize its existence in the more diluted solutions (from $\phi=6$ to $14 \%$ ) where no correlation maximum is observed in the scattering factor?

Moreover, the general picture of the transition summarized in point ii) can be qualitatively interpreted using a simple Landau approach : the free energy at rest is expanded in powers of the nematic order parameter $S$ (where $S^{2}, S^{3}$ and $S^{4}$ terms are taken into account) and a $\sigma^{2} S$ term is added to account for the quadratic coupling with the shear stress [22]. Indeed, such a model corresponds to a phase transition under a field (here $\sigma^{2}$ ) in the quasi-static limit. And the question arises of the validity of the quasi-static description far from equilibrium. Is the analogy of the shear-induced transition with a field-induced transition purely fortuitous or does it bear some deep physical meaning?

Lastly, the absence of any mechanical instability of shear banding type even far above $\sigma / G_{0}=2 / 3$ (point iii) for the $5 \%$ sample seems very puzzling. We should note however that the approach of Spenley et al. [7] was designed for the semidilute regime, i.e. the persistence length $\ell_{\mathrm{p}}$ is assumed to be much smaller than the Edwards correlation length $\xi$. On the other hand the $\mathrm{CPCl} / \mathrm{Sal}$ solution at $5 \%$ corresponds to the situation $\ell_{\mathrm{p}} \approx \xi$. May be this fact is sufficient to make the theory not appropriate to the present case. Another point in the SCM theory deserves a remark. The shear-banding instability described above is controlled by the first normal stress difference $N_{1}$ which is predicted to increase linearily with $\dot{\gamma}$. It would be therefore useful to complete the present data and measure $N_{1}$ both in the moderate and high $\phi$ ranges. Such investigations are currently under progress on the $\mathrm{CPCl} / \mathrm{Sal}$ solutions, but up to now we did not succeed in obtaining reliable data.

\section{Acknowledgments.}

We are indebted to R. Bruinsma and P. Linder for stimulating discussions.

\section{Appendix.}

The aim of this appendix is twofold : first to establish a relationship between the value of the transient shear stress $\sigma(t)$ and the volume fraction of the nematic phase $X_{\mathrm{N}} X(t)$ induced by the shearing at the same time $t$; second to find out the solution of equation (4) assuming this relation, and to show how it can be simplified into equation (6) under reasonable assumptions.

i) In the section devoted to the discussion of the nonlinear viscoelastic response of wormlike micellar solutions, it has been speculated that in the two-phase domain (where $\sigma(\dot{\gamma})=\sigma_{\mathrm{I} / \mathrm{N}}$ ), the sheared sample most likely exhibits a succession of (macroscopic) layers made from $\mathrm{L}_{1}$ and $\mathrm{N}_{\mathrm{c}}$ phases. Let us denote $\eta_{\mathrm{I}}$ and $\eta_{\mathrm{N}}$ their respective viscosities. Obviously, $\eta_{\mathrm{I}}>\eta_{\mathrm{N}}$. Since the stress is identical all through the sheared sample, each kind of layer is characterized 
by different shear rates called

$$
\dot{\gamma}_{1}=\frac{\sigma}{\eta_{1}}
$$

and

$$
\dot{\gamma}_{\mathrm{N}}=\frac{\sigma}{\eta_{\mathrm{N}}}
$$

In a controlled shear rate measurement, the overall shear rate $\dot{\gamma}$ is imposed by the experimental conditions, and so:

$$
\dot{\gamma}=\left(1-X_{\mathrm{N}} X(t)\right) \dot{\gamma}_{\mathrm{I}}+X_{\mathrm{N}} X(t) \dot{\gamma}_{\mathrm{N}}
$$

If we now replace the rates by their above equivalent given in equations (A.1) and (A.2), the shear stress at time $t$ reads :

$$
\sigma(t)=\frac{\eta_{1} \dot{\gamma}}{1+(\alpha-1) X_{\mathrm{N}} X(t)} \quad \text { with } \quad \alpha=\frac{\eta_{1}}{\eta_{\mathrm{N}}}
$$

Equation (A.4) depends on three parameters which are not directly experimentally accessible, the viscosity ratio $\alpha, \eta_{\mathrm{l}}$ and $X_{\mathrm{N}}$ (SANS measurements under shear should however provide a good estimate for the final volume fraction for $X_{\mathrm{N}}$ of nematic phase). In order to overcome this difficulty and to relate equation (A.4) more closely to the experimentally observed transient stresses, the boundary conditions are utilized. At $t=0, X(0)=0$ and thus $\sigma(0)=\eta_{\mathrm{I}} \dot{\gamma}$. As $t \rightarrow \infty, X(t)=1$ and thus :

$$
\sigma_{\mathrm{I} / \mathrm{N}}=\frac{\sigma(0)}{1+(\alpha-1) X_{\mathrm{N}}}
$$

From equations (A.4) and (A.5), one finally obtains the expression of the transient stress, as given by equation (2):

$$
\frac{\Delta \sigma(t)}{\sigma(t)}=\frac{\Delta \sigma(0)}{\sigma(0)}[1-X(t)]
$$

ii) If we now combine the last equation with equation (5) (which provides the differential equation in $X(t)$-variables), the time evolution of the shear stress in the metastable region is found to obey an implicit equation of the form :

$$
\frac{\sigma(0)}{\sigma_{\mathrm{I} / \mathrm{N}}} \ln \left[\frac{\sigma(0)\left(\sigma(t)-\sigma_{\mathrm{I} / \mathrm{N}}\right)}{\Delta \sigma_{0} \sigma(t)}\right]+\frac{\sigma(0)}{\sigma(t)}-1=-\frac{t^{2}}{\tau_{\mathrm{M}}^{2}} .
$$

Equation (A.7) unfortunately cannot be used to fit the experimental transient data with a standard fitting procedure. However, under some assumptions it can be simplified into a much more tractable expression. Actually, the $\sigma(t)$-function received from equation (A.7) depends on two parameters, $\tau_{\mathrm{M}}$ and $q=\Delta \sigma_{0} / \sigma_{\mathrm{I} / \mathrm{N}}$, which denotes the magnitude of the overshoot $\Delta \sigma_{0}$ relative to the value of the stress in the stationary limit. In figure A 1 the normalized stress overshoot $\Delta \sigma(t) / \Delta \sigma(0)$ has been plotted against time (with $\tau_{\mathrm{M}}=1 \mathrm{~s}$ ) for different values of $q$ $(q=0.1,0.5$ and $q=1)$.

As far as the $q$-parameter is small compared to unity (say $q<10 \%$ ), the transient behavior remains close to the $\exp \left(-t^{2}\right)$-evolution, also included in figure Al for comparison (open 


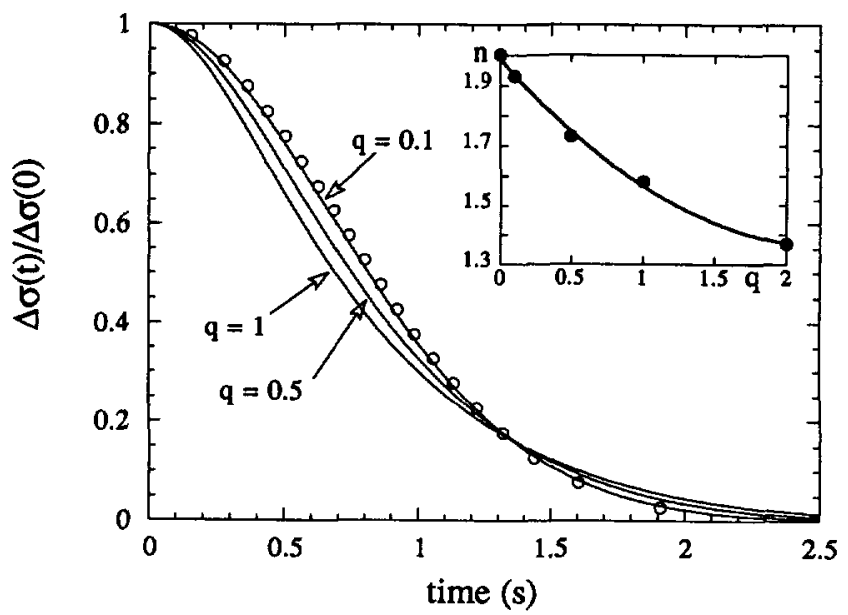

Fig. A1.

circles). For larger $q$-values $(q \sim 1), \sigma(t)$ deviates slightly from equation (6). However, the overall time behavior for the stress is preserved. The $\Delta \sigma(t) / \Delta \sigma(0)$-set of functions displayed in figure $\mathrm{A} 1$ has been found to be well accounted for by using a stretched exponential function of the form : $\Delta \sigma(t) / \Delta \sigma(0)=\exp \left(-\left(t / \tau_{M}\right)^{n}\right)$. In the inset of figure A1, the exponent $n$ is shown versus $q$. It shows that, as $q$ increases, $n$ decreases. Since in the present transient experiments the overshoot never exceeds $\sim 40 \%$ of the stationary limit value (see Tab. I for a compilation of typical transient data), $n$ lies in the range 1.7-2.

In conclusion of this appendix, and considering the above arguments, we can assume that the solutions can be approximated reasonably well by equation (6).

\section{References}

[1] For a review : Cates M. E. and Candau S. J., J Phys. Condens. Matter 2 (1990) 6869.

[2] Rehage H. and Hoffmann H., Mol. Phys. 74 (1991) 933 and references therein.

[3] ${ }^{\circ}$ Kern F., Zana R. and Candau S. J., Langmuir 7 (1991) 1344 ;

Khatory A., Lequeux F., Kern F. and Candau S. J., Langmuir (1993).

[4] Terech P., Schaffhauser V., Maldivi P. and Guenet J. M., Langmuir 8 (1992) 2104.

[5] Clausen T. M., Vinson P. K., Minter J. R., Davis H. T., Talmon Y. and Miller W. G., J. Phys. Chem. 96 (1992) 474.

[6] Berret J. F., Appell J. and Porte G., Langmuir 9 (1993) 2851.

[7] Spenley N. A., Cates M. E. and MacLeish T. C. B., submitted to Phys. Rev'. Lett (1993).

[8] Cates M. E., J. Phys. Chem. 94 (1990) 371.

[9] Doi M. and Edwards S. F., The Theory of Polymer Dynamics (Clarendon Press, Oxford, 1986).

[10] Cates M. E., Macromolecules 20 (1987) 2289 ; J. Phys. France 49 (1988) 1593.

[11] Granek R. and Cates M. E., J Chem. Phys. 96 (1992) 4758 and references therein.

[12] Schmitt V., Lequeux F.. Pousse A. and Roux D., preprint submitted to Langmurr (1993).

[13] Berret J.-F., Roux D. C., Porte G. and Lindner P., Europhys. Lett. 25 (1994) 521.

[14] El Haitamy O., Thèse de troisième cycle (1985), Université de Montpellier (France), unpublished.

[15] Larson R. G., Rheologica Acta 31 (1992) 497.

[16] Appell J., Bassereau P., Marignan J. and Porte G., Progr. Colloid. Polym. Sci 81 (1990) 13 ; Appell J. and Marignan J., J. Phys. II France 1 (1991) 1447. 
[17] Cabannes B., In Surfactant Solutions : New Methods of Investigation, R. Zana Ed. (Dekker, New York, 1993) p. 57.

[18] Hoffman H., Kalus J., Thurn H. and Ibel K., Ber. Bunsen Ges. Phys. Chem. 87 (1983) 1120.

[19] Ferry J. D., Viscoelastic Properties of Polymers (Wiley, New York, 1980).

[20] Actually, the persistence $\ell_{p}$ has not been measured yet for the present $\mathrm{CPCl} / \mathrm{Sal}$ system. Neutron scattering measurements are available for $\mathrm{CPBr} / \mathrm{NaBr}$ and $\mathrm{CPClO} 3 / \mathrm{NaClO}_{3}$ solutions.

[21] Descamps M., Galerne 1989 (Transitions de Phase dans les Solides), P. Gressier and P. Colombet Eds. (1989).

[22] Porte G. and Berret J.-F., in preparation. 